\title{
Proteomic profiling of non-obese type 2 diabetic skeletal muscle
}

\author{
EDEL MULLEN and KAY OHLENDIECK
}

Department of Biology, National University of Ireland, Maynooth, County Kildare, Ireland

Received October 22, 2009; Accepted November 24, 2009

DOI: 10.3892/ijmm_00000364

\begin{abstract}
Abnormal glucose handling has emerged as a major clinical problem in millions of diabetic patients worldwide. Insulin resistance affects especially one of the main target organs of this hormone, the skeletal musculature, making impaired glucose metabolism in contractile fibres a major feature of type 2 diabetes. High levels of circulating free fatty acids, an increased intramyocellular lipid content, impaired insulin-mediated glucose uptake, diminished mitochondrial functioning and an overall weakened metabolic flexibility are pathobiochemical hallmarks of diabetic skeletal muscles. In order to increase our cellular understanding of the molecular mechanisms that underlie this complex diabetesassociated skeletal muscle pathology, we initiated herein a mass spectrometry-based proteomic analysis of skeletal muscle preparations from the non-obese Goto-Kakizaki rat model of type 2 diabetes. Following staining of high-resolution two-dimensional gels with colloidal Coomassie Blue, 929 protein spots were detected, whereby 21 proteins showed a moderate differential expression pattern. Decreased proteins included carbonic anhydrase, 3-hydroxyisobutyrate dehydrogenase and enolase. Increased proteins were identified as monoglyceride lipase, adenylate kinase, $\mathrm{Cu} / \mathrm{Zn}$ superoxide dismutase, phosphoglucomutase, aldolase, isocitrate dehydrogenase, cytochrome c oxidase, small heat shock Hsp27/B1, actin and 3-mercaptopyruvate sulfurtransferase. These proteomic findings suggest that the diabetic phenotype is associated with a generally perturbed protein expression pattern, affecting especially glucose, fatty acid, nucleotide and amino acid metabolism, as well as the contractile apparatus, the cellular stress response, the anti-oxidant defense system and detoxification mechanisms. The altered expression levels of distinct skeletal muscle proteins, as documented in this study, might be helpful for the future establishment of a
\end{abstract}

Correspondence to: Professor Kay Ohlendieck, Department of Biology, National University of Ireland, Maynooth, Co. Kildare, Ireland

E-mail: kay.ohlendieck@nuim.ie

Abbreviations: CCB, colloidal Coomassie Blue; GK, GotoKakazaki; MS, mass spectrometry; RuBPs, ruthenium II tris bathophenanthroline disulfonate

Key words: carbonic anhydrase, Goto-Kakazaki, monoglyceride lipase, muscle proteomics, type 2 diabetes comprehensive biomarker signature of type 2 diabetes. Reliable markers could be used for improving diagnostics, monitoring of disease progression and therapeutic evaluations.

\section{Introduction}

The number of diabetic patients suffering from severe metabolic disturbances and glucotoxic complications is rapidly increasing worldwide and the incidence of diabetes is approaching endemic proportions (1). Type 2 diabetes mellitus represents a highly complex and heterogeneous disease that is influenced by both genetic and environmental factors (2). A modern sedentary lifestyle and obesity-associated metabolic complications clearly play an essential role in disease progression (3). The principal features of type 2 diabetes are an abnormal sensitivity of peripheral tissues to insulin and decreased levels of hormone secretion. Insulin resistance affects especially skeletal muscles, since contractile fibres are responsible for most of the insulin-triggered whole body glucose disposal. This makes impaired insulin signaling and disturbed glucose metabolism in muscle tissues a striking feature of type 2 diabetes (4).

In contrast to the well-established cardiomyopathic pathology of diabetes (5), it is poorly understood how abnormal tissue sensitivity to insulin causes decreased skeletal muscle strength (6), especially in older individuals with type 2 diabetes (7). Besides impaired insulin-mediated glucose uptake, fatty acid metabolism also has a profound influence on diabetic side effects. Type 2 diabetes is associated with high levels of circulating free fatty acids, an increased intramyocellular lipid content, diminished mitochondrial functioning and an overall weakened metabolic flexibility in the skeletal musculature, as reviewed by Phielix and Mensink (8). Lipid deposition in non-adipose tissues, such as skeletal muscle, has been shown to be related to insulin resistance, whereby possibly the accumulation of triacylglycerol does not directly influence insulin signaling but indirectly via its lipid metabolites (9).

In order to further our molecular understanding of diabetesrelated abnormalities in skeletal muscles, mass spectrometrybased proteomics suggests itself as an ideal analytical tool for performing global screening approaches to determine potential alterations in protein expression levels (10). As recently reviewed by Sundsten and Ortsaeter (11), numerous proteomic programmes have been initiated to unravel the complex pathobiochemical mechanisms that underlie diabetes, focusing especially on the pancreas, blood, adipose tissue and the liver. In analogy, here we carried out the proteomic profiling of crude skeletal muscle extracts from the Goto-Kakizaki 
(GK) rat model of type 2 diabetes (12). GK rats are spontaneously diabetic animals that exhibit chronically impaired insulin signaling $(13,14)$, which usually occurs by 4 weeks of age (15). They are characterized by increased blood glucose levels without significant alterations in non-fasting plasma insulin levels (16). Diabetic GK skeletal muscles are characterized by a diminished recruitment of the glucose transporter isoform GLUT4 (17), membrane cytoskeletal defects in the dystrophin-dystroglycan complex $(17,18)$, an inhibition of insulin receptor auto-phosphorylation (19), impaired activities of insulin signaling intermediates (20), abnormal mitochondrial functioning (21) and a reduced percentage of oxidative fibres (22). Since diabetic GK rats are non-obese, fundamental mechanisms of type 2 diabetes can be investigated without potentially complicating obesityrelated factors.

The proteomic analysis of normal versus non-obese diabetic skeletal muscles presented herein has revealed a moderate differential expression pattern for 21 proteins, whereby 7 proteins were found to be reduced and 14 proteins to be increased in their abundance in GK tissue. With respect to neuromuscular disorders, the mass spectrometric cataloguing of normal muscle and the profiling of genetic and physiological animal models over the last few years have established large proteomic maps (23). Databanks of biomarkers that are implicated in muscular atrophy, fibre transformation, muscular dystrophy or age-related muscle wasting are now available for comparative biochemical studies (24). In this respect, the proteomic findings of this study agree with the idea that diabetes mellitus is associated with a generally perturbed protein expression pattern. Insulin resistance appears to be closely related to abnormalities in glucose, fatty acid, nucleotide and amino acid metabolism, as well as changes in the contractile apparatus, the anti-oxidant defense system, detoxification mechanisms and the cellular stress response.

\section{Materials and methods}

Materials. For the comparative gel electrophoretic analysis of normal versus diabetic muscle proteins, Imobiline $\mathrm{pH}$ gradient IPG dry strips, ampholytes, cover fluid and acetonitrile were purchased from Amersham Bioscience/GE Healthcare (Little Chalfont, Bucks, UK). Ultrapure Protogel acrylamide stock solutions were obtained from National Diagnostics (Atlanta, GA, USA). Gel electrophoretic buffer systems, protein molecular weight ladders and protein assay reagents were purchased from BioRad Laboratories (Hemel-Hempstead, Hertfordshire, UK). For the peptide mass spectrometric identification of muscle proteins, sequencing grade-modified trypsin was purchased from Promega (Madison, WI, USA). LC-MS Chromasolv water and formic acid were from Fluka (Milwaukee, WI, USA). For the visualization of gel electrohoretically separated proteins, Coomassie Brilliant Blue G-250 dye was purchased from Thermo Fisher Scientific (Waltham, MA, USA). Protease inhibitors were from Roche Diagnostics (Mannheim, Germany). All other analytical-grade chemicals were purchased from Sigma Chemical Company (Dorset, UK).

Animal model. As an internationally established animal model of type 2 diabetes, the spontaneous diabetic GK rat (12) was used in this study. In obesity-related diabetes, high levels of circulating free fatty acids and an extensive intramyocellular accumulation of triacylglycerol probably play a key role in causing decreased tissue sensitivity for insulin. However, since the GK rat is non-obese, the analysis presented herein eliminates to a large extent complicating factors due to excess lipids in muscle fibres and focuses instead on the core defects in a spontaneous form of type 2 diabetes. Rats were purchased from M\&B Taconic Ltd. Animal Suppliers (Ry, Denmark). For comparative proteomic studies, the accessible protein complement was extracted from freshly dissected gastrocnemius muscles from 9-week-old normal Wistar rats and age-matched GK rats. The validation of the diabetic status of the cohort of GK rats used in this study has previously been documented (17). Non-fasting blood samples from GK rats showed a significant increase in glucose levels as compared to Wistar rats, but plasma insulin levels were relatively comparable between both rat strains. The average body weight of diabetic animals was found to be slightly below that of normal rats.

Preparation of total gastrocnemius muscle extracts. Muscle samples with a wet weight of $200 \mathrm{mg}$ were quick-frozen in liquid nitrogen and ground into a fine powder using a mortar and pestle. The muscle powder was subsequently placed into $1 \mathrm{ml}$ lysis buffer (7 M urea, $2 \mathrm{M}$ thiourea, 4\% (w/v) CHAPS, $100 \mathrm{mM}$ DTT and 2\% (v/v) pH 3-10 ampholytes), which was supplemented with a protease inhibitor cocktail (25). Following incubation at room temperature for $3 \mathrm{~h}$, the suspension was centrifuged at $14,000 \mathrm{x} \mathrm{g}$ for $20 \mathrm{~min}$ at $4^{\circ} \mathrm{C}$. The total protein complement extracted from normal and diabetic tissues was quantified by the Bradford method (26).

Two-dimensional gel electrophoresis. Total muscle extracts from normal and diabetic muscle tissues were separated in the first dimension by isoelectric focusing and in the second dimension by sodium dodecyl sulphate polyacrylamide gel electrophoresis, as previously described $(27,28)$. For the comparative proteomic analysis, 4 biological repeats of normal and 4 biological repeats of diabetic samples was used. Isoelectric focusing strips were rehydrated in rehydration buffer [7 $\mathrm{M}$ urea, $2 \mathrm{M}$ thiourea, 4\% (w/v) CHAPS, 1.2\% deStreak and $2 \%(\mathrm{v} / \mathrm{v}) \mathrm{pH} 3-10$ ampholytes] and $700 \mathrm{mg}$ of muscle protein sample for $12 \mathrm{~h}$. First-dimension protein separation was carried out on an Amersham IPGphor IEF system, following the manufacturer's recommendations and the optimized protocol of our laboratory for the separation of skeletal muscle proteins (28). Following chemical reduction and alkylation by a standardized protocol (25), first-dimension strips were carefully placed on top of $12.5 \%$ (w/v) slab gels and electrophoresed in an Amersham Ettan DALT-Twelve system at $1.5 \mathrm{~W}$ per gel until the bromophenol blue dye front had just ran off the gel. The protein separation pattern on twodimensional gels was visualized by colloidal Coomassie Blue (29), silver (30) or fluorescent RuBPs (31) staining. Highresolution gel images where analysed with the Progensis software programme from Non-Linear Dynamics (Newcastle upon Tyne, UK).

Mass spectrometric identification of muscle proteins. In order to unequivocally identify proteins of interest, the peptide mass 
Normal muscle

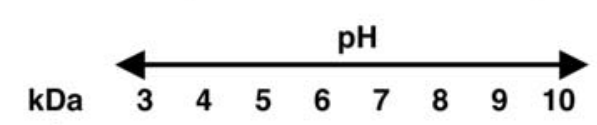

$97-$
49
35
22
16

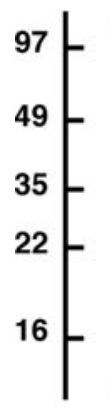

97 -
49
35
22
16
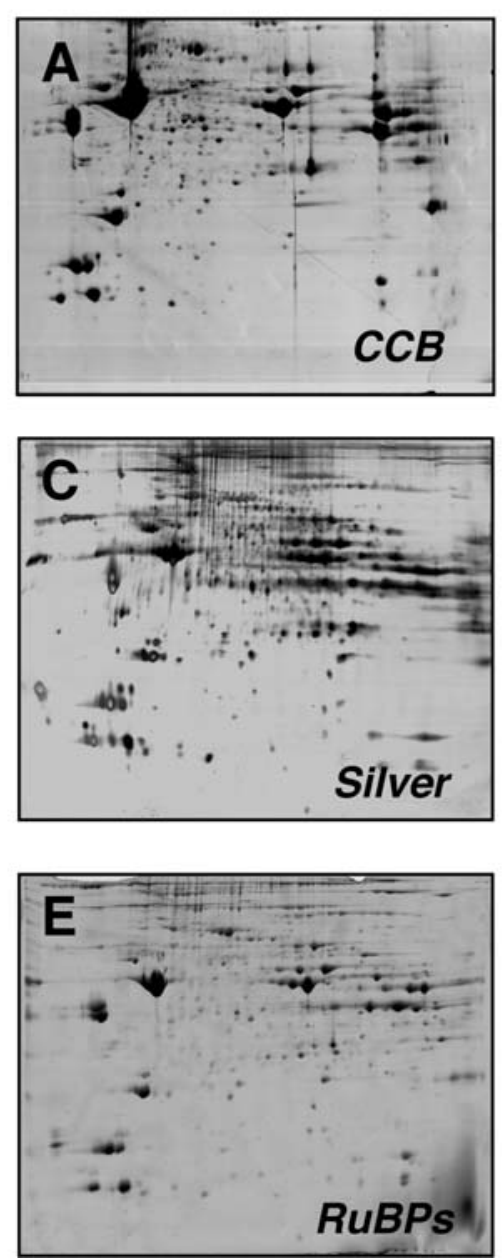

\section{Diabetic muscle}
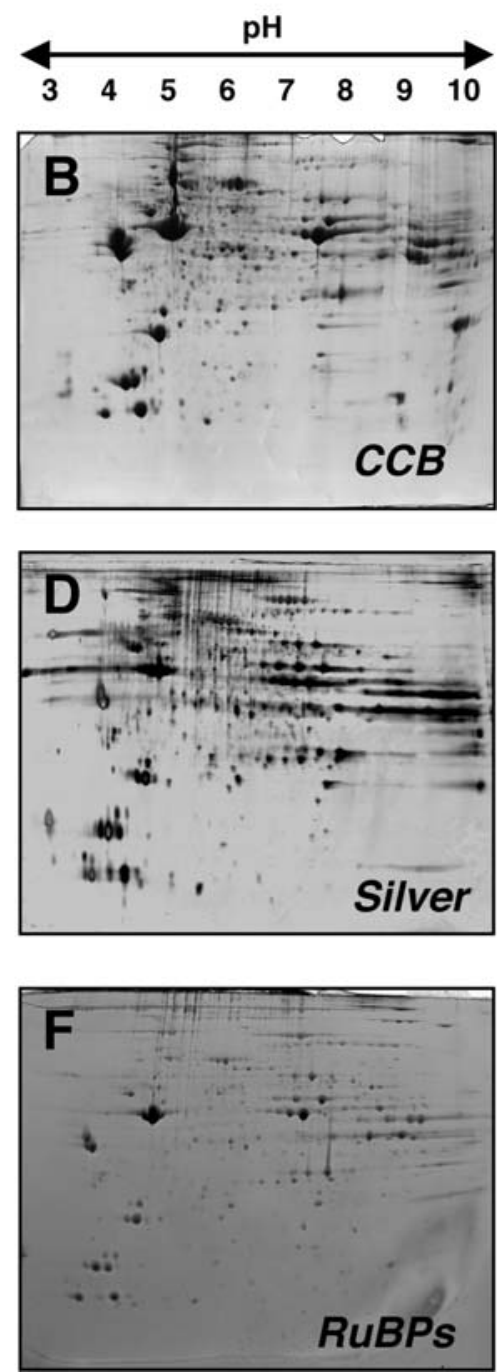

Figure 1. Comparative two-dimensional gel electrophoretic analysis of normal versus diabetic rat skeletal muscle. Crude total muscle extracts from normal and Goto-Kakizaki (GK) muscle tissue were separated in the first dimension by isolelectric focusing and in the second dimension by sodium dodecyl sulfate polyacrylamide gel electrophoresis. Shown are colloidal Coomassie Blue (CCB; A and B), silver (C and D) or fluorescent Ruthenium II bathophenanthroline disulfonate chelate (RuBPs; E and F) stained gels of normal (A, C and E) versus diabetic GK muscle (B, D and F). The pH-values of the first dimension gel system and molecular mass standards (in $\mathrm{kDa}$ ) of the second dimension are indicated on the top and on the left of panels, respectively.

spectrometric analysis of muscle proteins with a changed abundance in diabetic muscle tissue was carried out on a Model 6430 Ion Trap LC/MS apparatus from Agilent Technologies (Santa Clara, CA, USA). Excision, washing, destaining and treatment with trypsin were performed by a previously optimised method (28). Trypsin-generated peptides were obtained by removing supernatants from digested gel plugs. Further recovery was achieved by adding 30\% acetonitrile/ $0.2 \%$ trifluoroacetic acid to the gel plugs for $10 \mathrm{~min}$ at $37^{\circ} \mathrm{C}$ with gentle agitation. Resulting supernatants were pooled with the initially recovered cohort of peptides following trypsin digestion.

Further peptide recovery was achieved through the addition of $60 \%$ acetonitrile/ $0.2 \%$ trifluoric acid to each plug for $10 \mathrm{~min}$ at $37^{\circ} \mathrm{C}$ with gentle agitation. Supernatants were added to the peptide pool. The sample was dried through vacuum centrifugation and the concentrated peptide fractions were then resuspended in mass spectrometry-grade distilled water and
$0.1 \%$ formic acid for identification by ion trap LC-MS analysis. Separation of peptides was performed with a nanoflow Agilent 1200 series system, equipped with a Zorbax 300SB C18 $5 \mu \mathrm{m}$, $4 \mathrm{~mm} 40 \mathrm{nl}$ pre-column and an Zorbax 300SB C18 $5 \mu \mathrm{m}$, $43 \mathrm{~mm} \times 75 \mu \mathrm{m}$ analytical reversed phase column using the HPLC-Chip technology (32).

Mobile phases utilized were A: $0.1 \%$ formic acid, B: $50 \%$ acetonitrile and $0.1 \%$ formic acid. Samples were loaded into the enrichment at a capillary flow rate set to $2 \mu \mathrm{l} / \mathrm{min}$ with a mix of $\mathrm{A}$ and $\mathrm{B}$ at a ratio 19:1. Tryptic peptide fragments were eluted with a linear gradient of $10-90 \%$ solvent B over $2 \mu \mathrm{l} /$ min with a constant nano pump flow of $0.6 \mathrm{ml} / \mathrm{min}$. A $1 \mathrm{~min}$ post-time of solvent A was used to remove sample carry over. The capillary voltage was set to $1700 \mathrm{~V}$. The flow and the temperature of the drying gas were $4 \mathrm{1} / \mathrm{min}$ and $300^{\circ} \mathrm{C}$, respectively (33). Database searches were carried out with Mascot MS/MS Ion search (Matrix Science, London, UK; MSDB database, release 20063108). All pI values and 


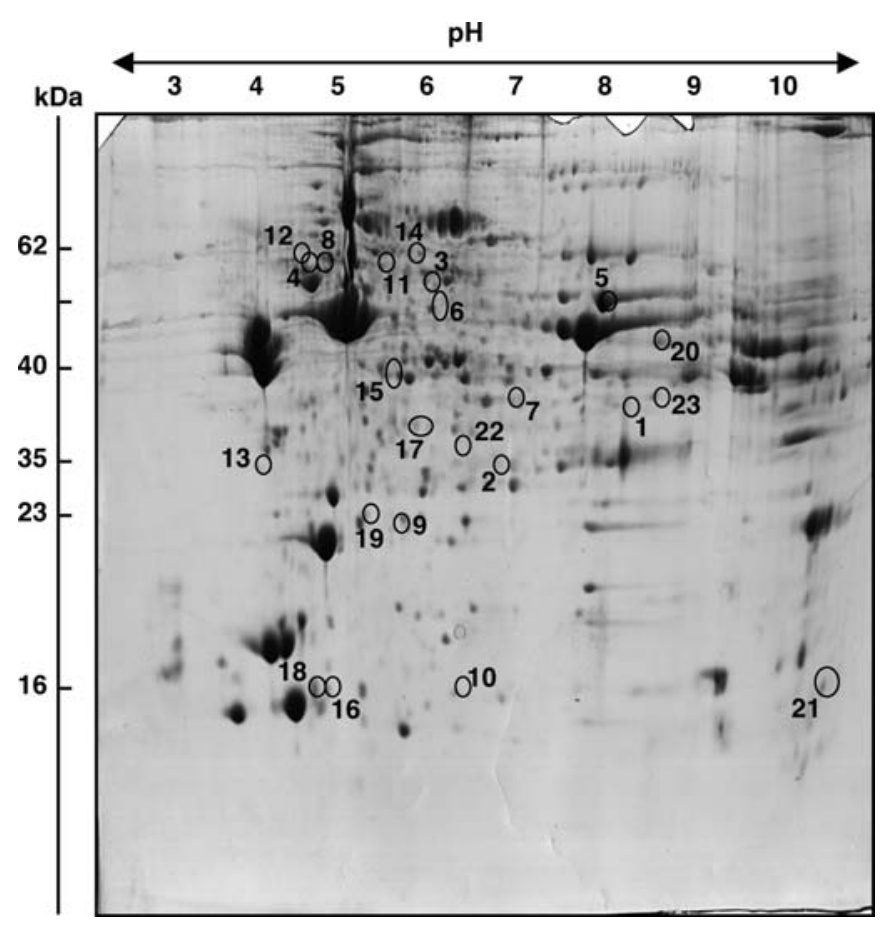

Figure 2. Two-dimensional reference gel of diabetic rat skeletal muscle. Shown is a colloidal Coomassie Blue stained reference gel of diabetic rat skeletal muscle, used for the mass spectrometric identification of proteins with a differential expression profile. The $\mathrm{pH}$-values of the first dimension gel system and molecular mass standards (in $\mathrm{kDa}$ ) of the second dimension are indicated on the top and on the left of the panel, respectively. Identified muscle proteins are marked by circles and are numbered 1 to 23. See Table I for a detailed listing of proteins that exhibit a diabetes-associated change in their abundance.

molecular masses of identified muscle proteins were compared to the relative position of their corresponding two-dimensional spots on analytical slab gels.

\section{Results}

Comparative gel electrophoretic analysis of normal versus diabetic skeletal muscle. In order to determine potential differences in the skeletal muscle proteome from normal Wistar rats versus diabetic GK rats, crude total tissue extracts were separated by high-resolution two-dimensional gel electrophoresis. The gastrocnemius muscle with a mixed fibre type was chosen for the initial proteomic survey of diabetic effects on the muscle protein complement, since comparative data from numerous proteomic studies exist with respect to this muscle (34-37). Fig. 1 shows representative gels of normal versus diabetic muscle preparations stained with $\mathrm{CCB}$, silver or fluorescent RuBPs. The high-resolution gels contained 929, 1236 and 1561 detectable spots in CBB, silver and RuBPs-labelled gels, respectively. In general, the twodimensional spot pattern of the normal muscle protein complement was found to be in agreement with previously published studies on the gel electrophoretic separation of total skeletal muscle extracts (38-40). The protein spot distribution of normal versus diabetic preparations did not show extensive differences, but densitometric scanning revealed moderate alterations in distinct classes of muscle proteins.
Densitometric analysis of normal versus diabetic skeletal muscle. Analytical two-dimensional gels were stained with colloidal Coomassie Blue and images from normal versus diabetic preparations compared with the help of a Typhoon Trio variable imager and Progenesis 2-D analysis software. Fig. 2 shows a reference gel of diabetic rat skeletal muscle used for the mass spectrometric identification of proteins with a differential expression profile. Muscle proteins with a changed abundance are marked by circles and are numbered 1-23. Protein species with a changed abundance in GK gastrocnemius muscle ranged in molecular mass from $15.9 \mathrm{kDa}$ $(\mathrm{Cu} / \mathrm{Zn}$ superoxide dismutase) to $67.6 \mathrm{kDa}$ (dihydrolipoamide s-acetyltransferase of the pyruvate dehydrogenase complex) and covered a $\mathrm{p} I$-range from $\mathrm{p} I 4.9$ (ATP synthase) to $\mathrm{p} I 8.9$ (ß-globin). A decreased expression was found in the case of 7 muscle proteins, and 16 proteins were shown to be increased in their abundance.

Mass spectrometric identification of proteins with a diabetesrelated change in abundance. ESI MS analysis was used to unequivocally identify protein species contained in twodimensional spots with an altered density in normal versus diabetic preparations. A list of the 23 muscle-associated proteins that exhibited a significantly altered expression level in GK muscle is shown in Table I. The table summarizes CCB-stained proteins separated in the $\mathrm{pH}$ 3-10 range and outlines matched peptide sequences, percentage sequence coverage, Mascot score, the relative molecular mass, $\mathrm{pI}$-value, protein accession number and fold-change of individual muscle proteins affected by the diabetic phenotype.

The spot numbers of MS-identified protein species listed in Table I correlate with the numbering of two-dimensional spots marked in Fig. 2. The majority of identified muscle proteins were found to be constituents of various metabolic pathways. This included enzymes and transporters involved in glycolysis, the citric acid cycle, oxidative phosphorylation, lipolytic catabolism, nucleotide metabolism, carbon dioxide removal, oxygen transportation, and amino acid catabolism. In addition, components of the contractile apparatus, the cellular stress response, anti-oxidant defense mechanisms and formaldehyde detoxification appear to be affected in diabetic muscle tissue.

Skeletal muscle proteins with diabetes-associated expression changes. The two muscle protein species with the highest fold decrease were identified as carbonic anhydrase isoform CA3 (spot 1) and 3-hydroxyisobutyrate dehydrogenase (spot 2). Other proteins with reduced expression level were found to be the E2 component of the 2-oxo-glutarate dehydrogenase complex (spot 3), enolase (spots 5 and 7) and esterase D formylglutathione hydrolase (spot 6). Spots 4 and 8 , which were both identified as mitochondrial ATP synthase, exhibited decreased and increased levels, respectively. These findings are difficult to interpret, but may be due to differential pathological effects on post-translational modifications in ATP synthase molecules. This might explain the opposite alterations in expression levels of two ATP synthase isoforms with differing isoelectric points and molecular masses.

Muscle-associated proteins with an increased abundance in GK muscle preparations were identified as adenylate kinase 


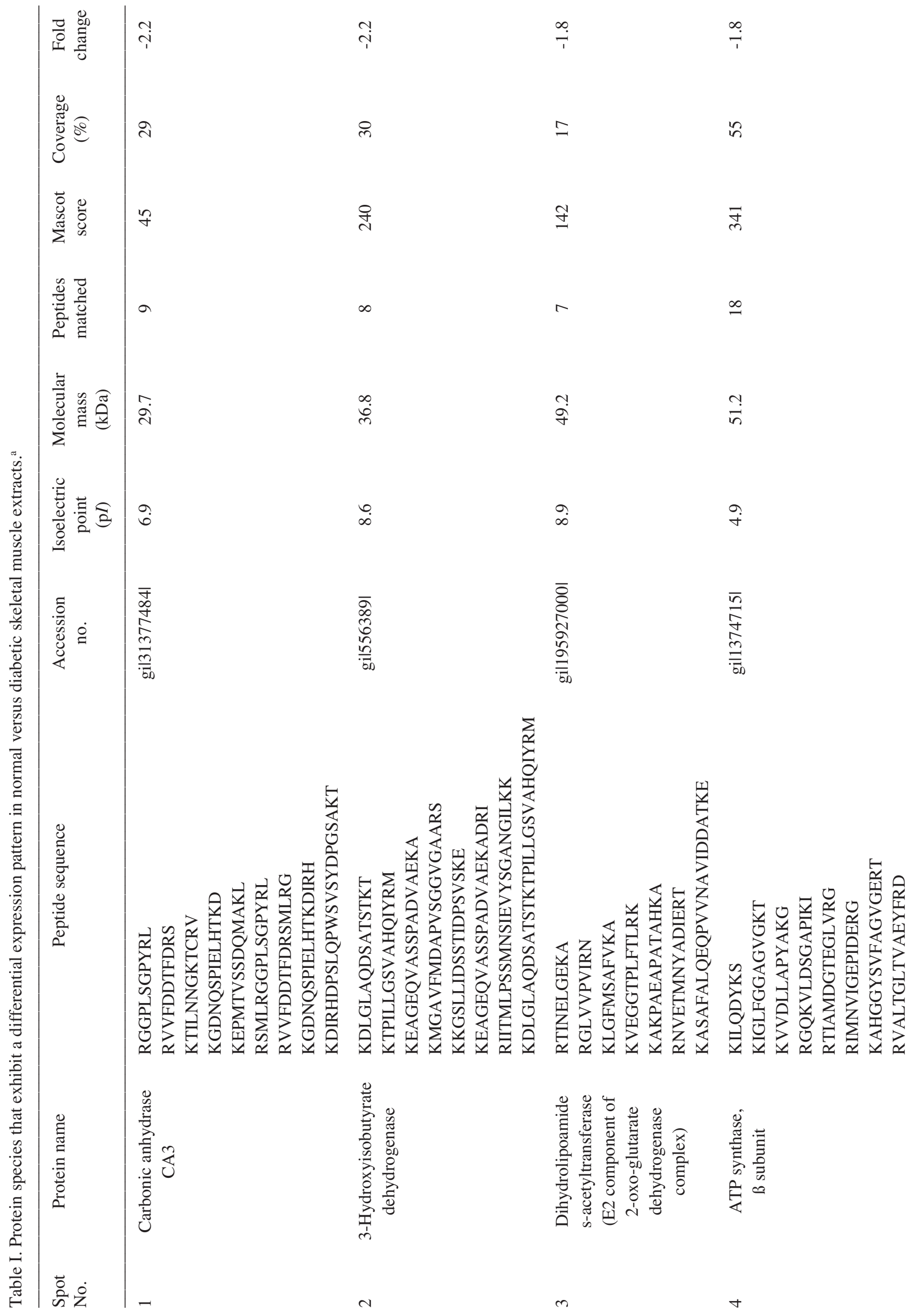



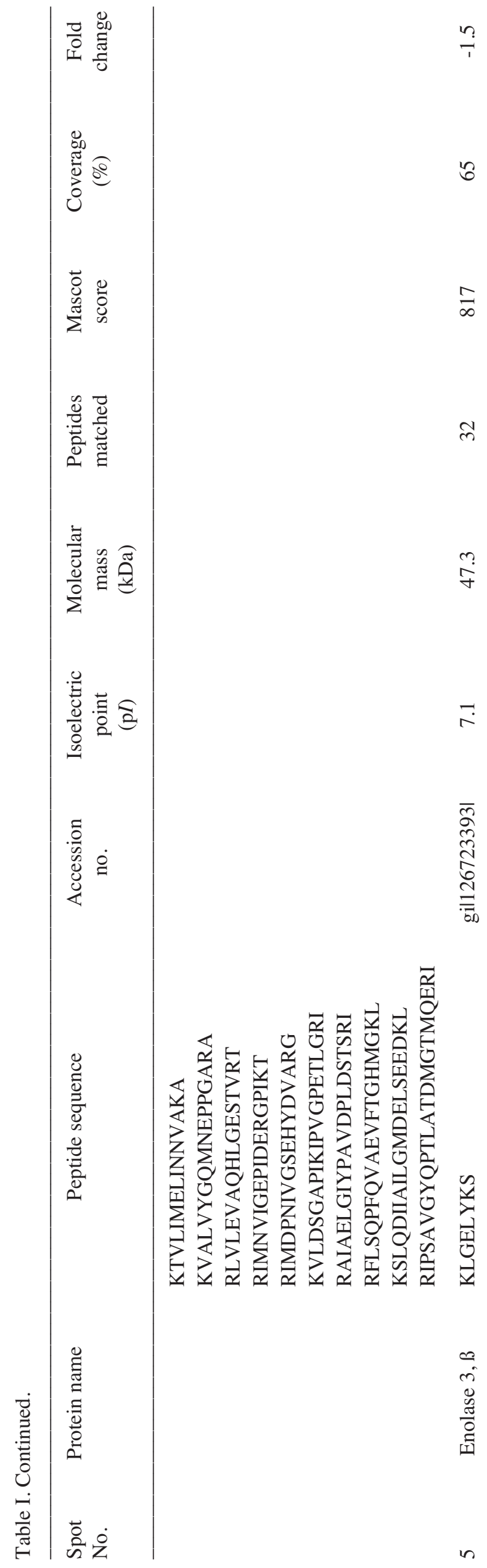

$\frac{1}{\infty}$

กี

$\stackrel{?}{q}$

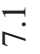

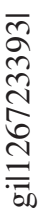

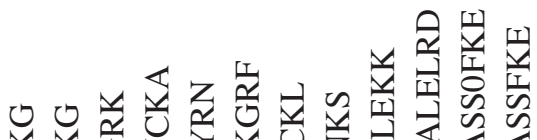

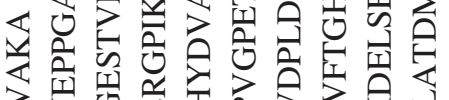

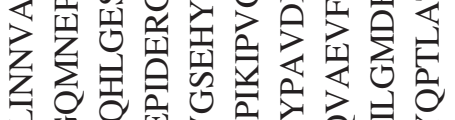

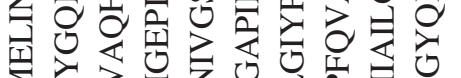

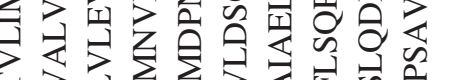

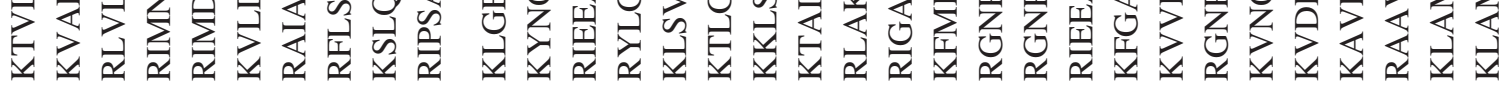

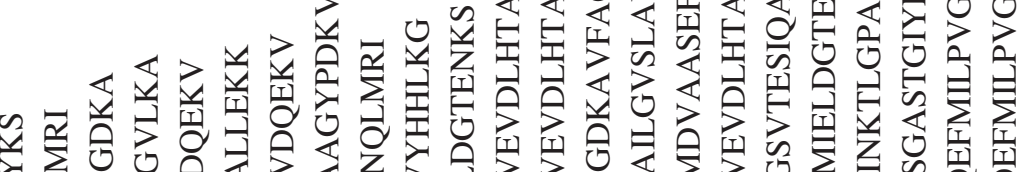

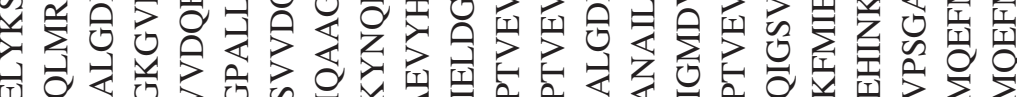

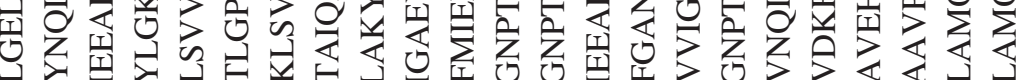




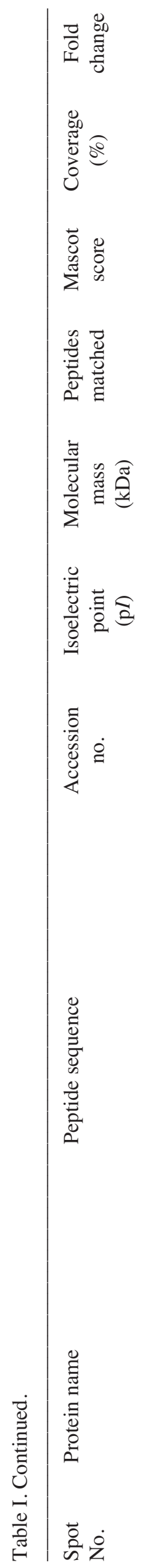

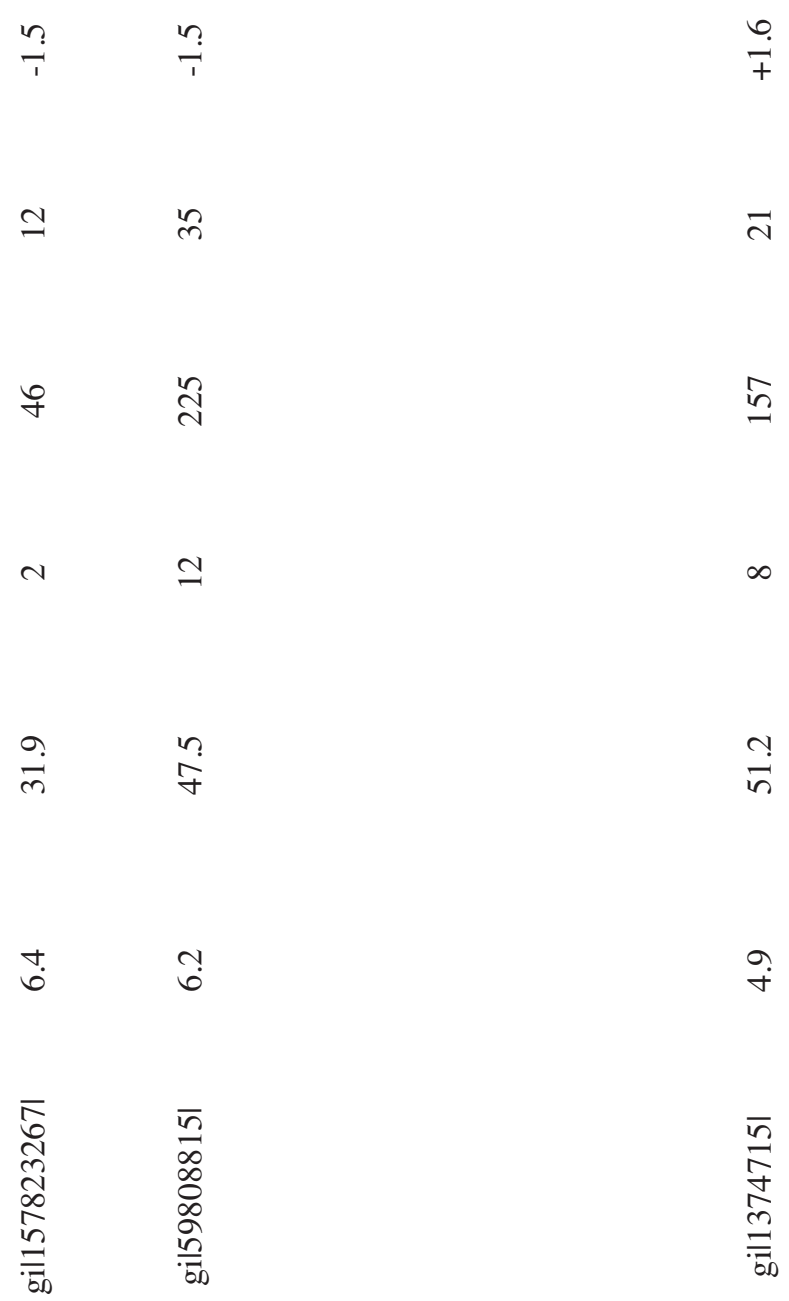
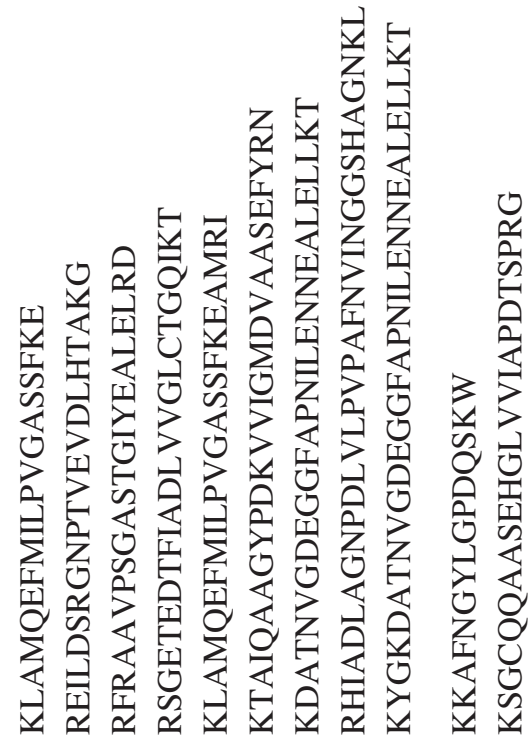

(1)

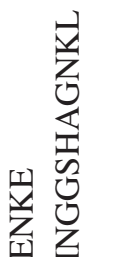

필

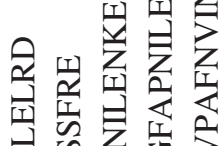

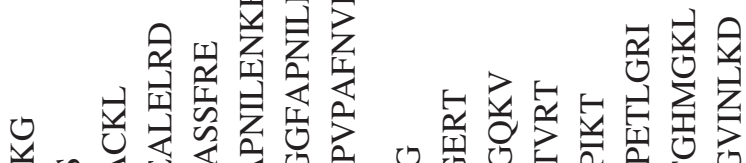

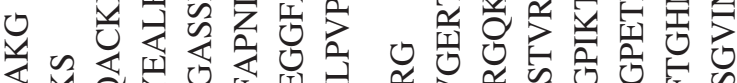

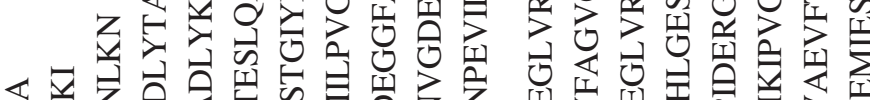

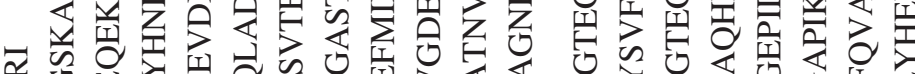

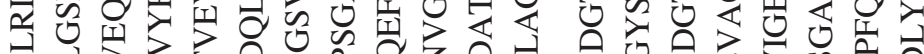

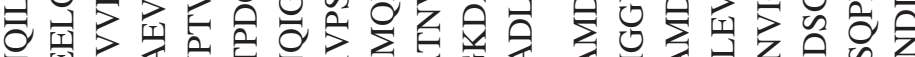

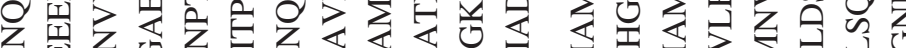

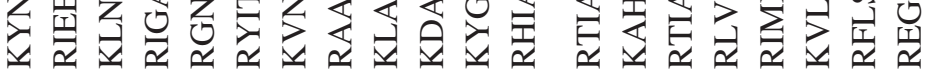

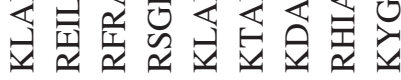
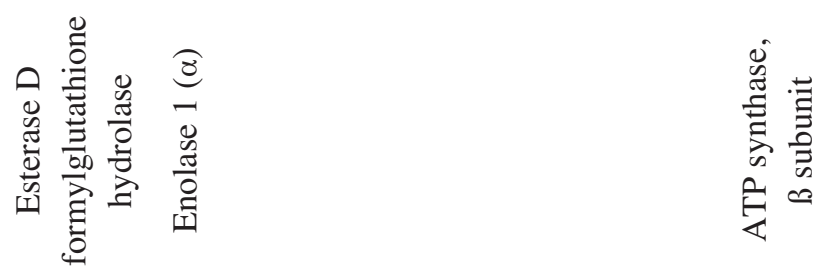


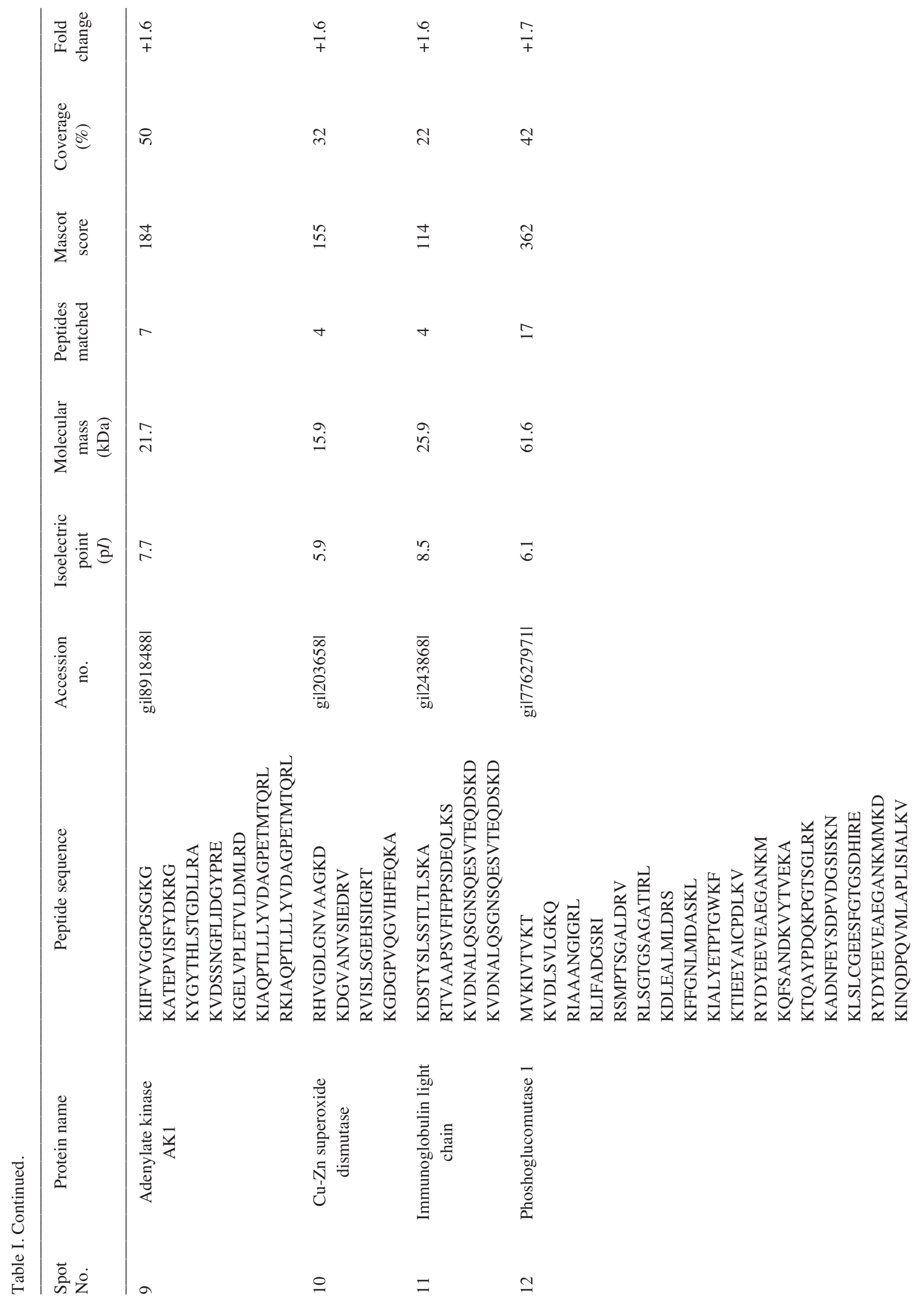




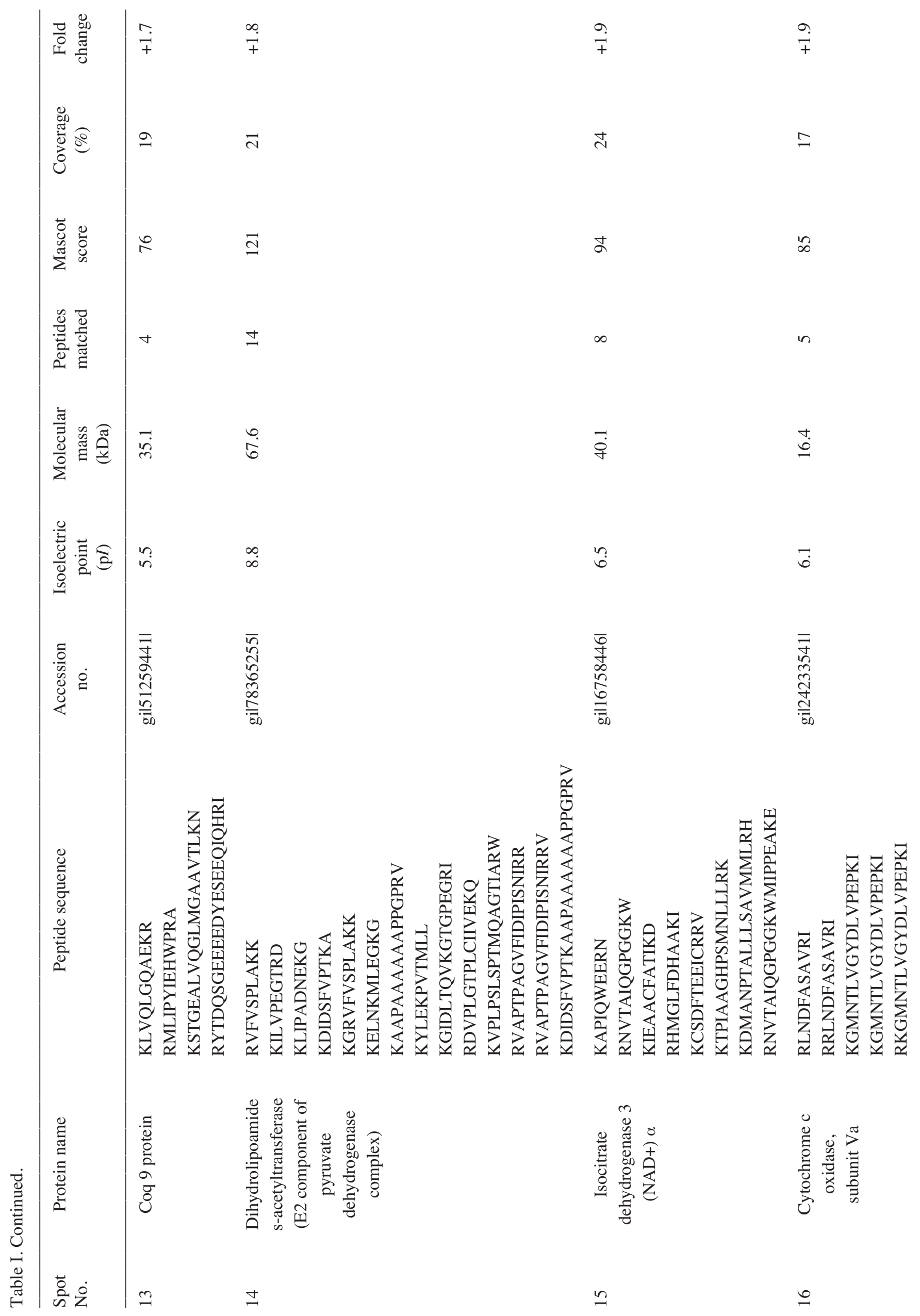




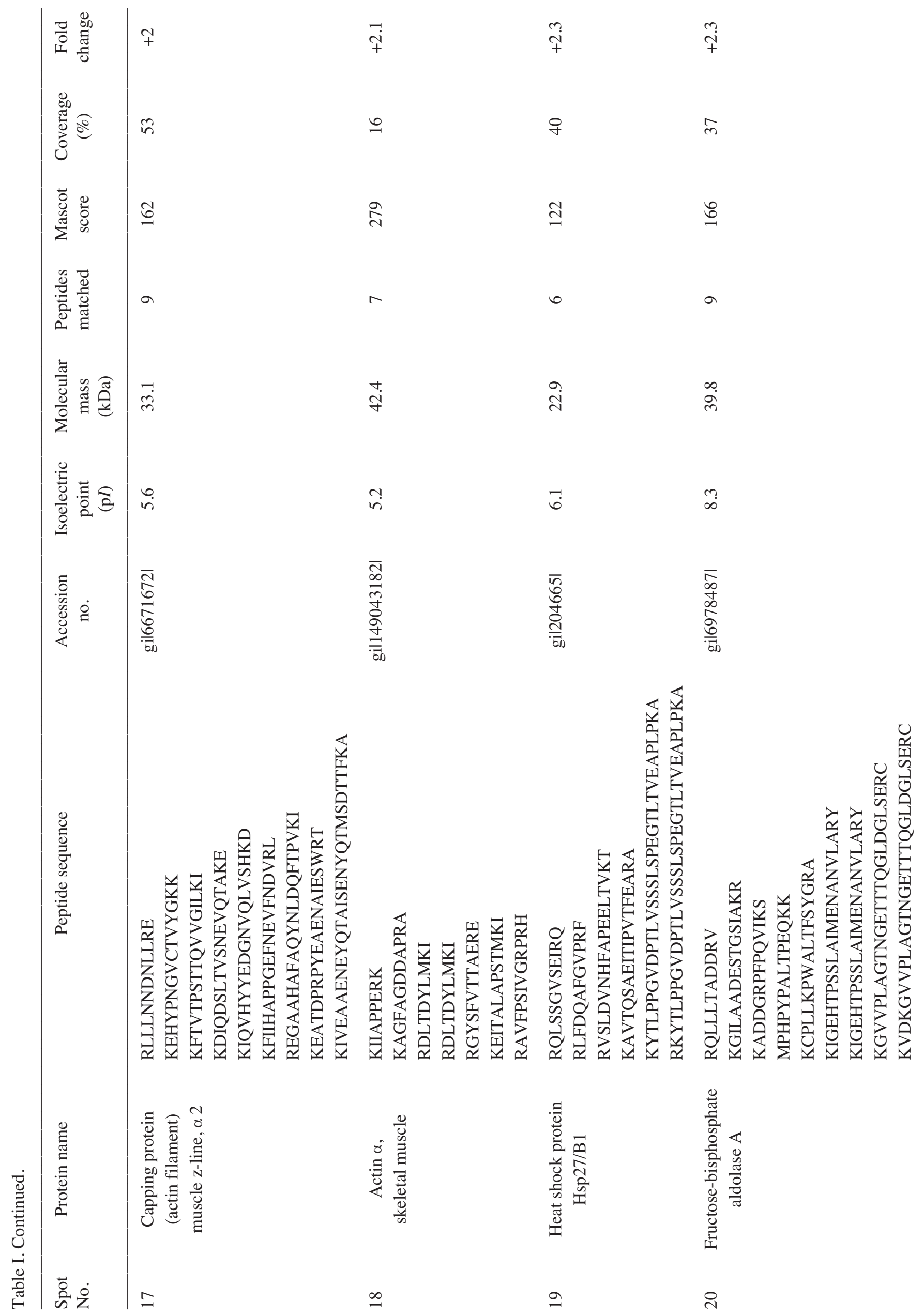




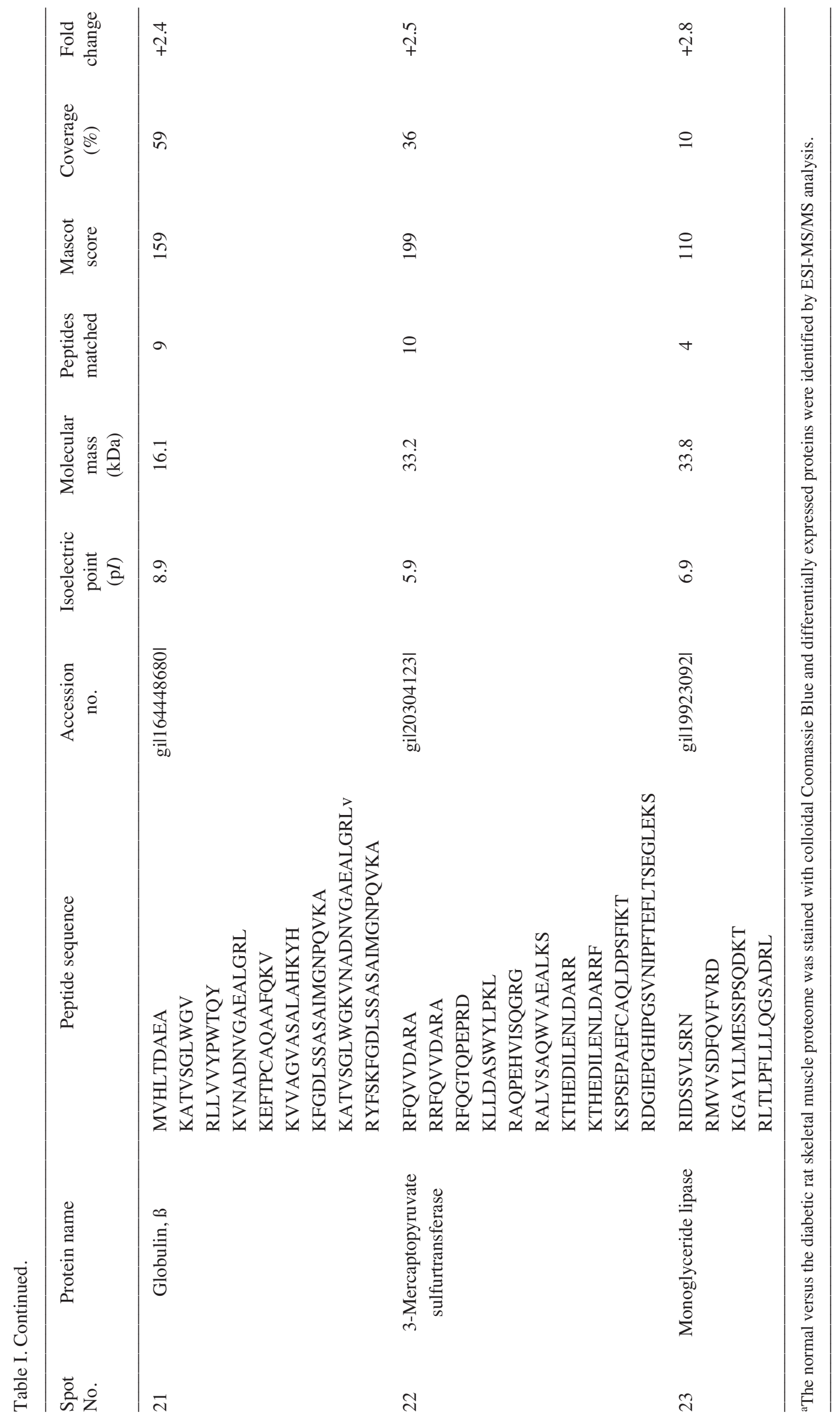


isoform AK1 (spot 9), Cu/Zn superoxide dismutase (spot 10), immunoglobulin light chain (spot 11), phosphoglucomutase 9 (spot 12), Coq 9 protein (spot 13), the E2 component of the pyruvate dehydrogenase complex (spot 14), isocitrate dehydrogenase (spot 15), cytochrome c oxidase (spot 16), capping protein of the actin filament (spot 17), $\alpha$-actin (spot 18), stress protein Hsp27/B1 (spot 19), fructose-biphosphate aldolase (spot 20), B-globulin (spot 21), and 3-mercaptopyruvate sulfurtransferase (spot 22). Interestingly, the muscle protein species with the highest fold increase was identified as the enzyme monoglyceride lipase (spot 23).

Silver-and RuBPs-stained protein candidates with diabetesassociated expression changes. Following the detailed MSbased identification of CBB-stained proteins, diabetes-related changes in the expression of silver- or fluorescent RuBPslabelled proteins was carried out. Although larger numbers of total protein spots were visualized by these two methods, surprisingly no additional components with markedly higher fold changes were identified. Interesting silver-stained proteins with a lower abundance in GK muscle were found to be acylCoA dehygrogenase (gil56541110l; pI 8.9; $70.8 \mathrm{kDa}$ ) and pyruvate kinase (gil1675994l; pI 6.6; $57.8 \mathrm{kDa}$ ). Fluorescent RuBPs staining revealed reduced expression of malate dehydrogenase isoform MDH-1 (gil37590235I; pI 6.1; $36.5 \mathrm{kDa}$ ) in diabetic tissue preparations.

\section{Discussion}

Diabetes mellitus and its associated complications affect millions of patients worldwide, causing blindness, cardiomyopathy, kidney failure, stroke and skeletal muscular weakness (41). Older adults with type 2 diabetes exhibit significantly decreased muscle strength (7), which is thought to be a major contributor to the development of physical disability in the senescent population (42). These clinical facts clearly warrant detailed biochemical investigations into the molecular and cellular mechanisms that underlie abnormal hormone signalling in diabetic muscle tissue. Insulin resistance in peripheral organ systems, representing one of the main features of diabetes-related dysregulation, is believed to be already present at a very early stage of the pre-diabetic state (10).

In early type 2 diabetes, low levels of insulin resistance can probably be partially compensated by increased secretion levels via enhanced pancreatic $\beta$-cell activity. However, at more advanced stages of diabetes, $B$-cell failure occurs leading to inadequate amounts of circulating insulin to overcome defects in tissue sensitivity (8). Since type 2 diabetes is an extremely complex and heterogeneous disorder (2), and probably involves both genetic and environmental factors, an unbiased global analysis of diabetic tissues by proteomics should be useful to identify novel indicators of its molecular pathogenesis. In this respect, the mass spectrometry-based proteomic survey of diabetic skeletal muscle tissue presented herein has successfully revealed a variety of expression changes in key muscle proteins. However, as compared to more severe muscular defects $(23,24)$, the diabetes-related expression changes of metabolic enzymes is relatively moderate.
The proteomic analysis of GK muscles indicates that abundant enzymes, transporters and structural components are affected in the non-obese diabetic phenotype. This includes muscle-associated proteins involved in glycolysis, the citric acid cycle, oxidative phosphorylation, lipolytic catabolism, nucleotide metabolism, carbon dioxide removal, oxygen transportation, amino acid catabolism, the contractile apparatus, cellular detoxification mechanisms and the stress response. The muscle protein species with the highest fold decrease were identified as 3-hydroxy-isobutyrate dehydrogenase and carbonic anhydrase CA3. Hydroxy-isobutyrate dehydrogenase is involved in a rate-limiting step of the degradation of valine, leucine and isoleucine (43). The resulting carbon skeleton can be utilized as a metabolic substrate for the generation of energy, and this mechanism appears to be weakened in diabetic muscle tissues. Interestingly, the $\mathrm{CO}_{2}$-removal mechanism and a specific detoxification mechanism seem to be affected in GK muscles. Since the muscle-specific isoform CA3 of carbonic anhydrase catalyses the vital conversion of $\mathrm{CO}_{2}$ into carbonic acid (44), its reduced expression suggests an impaired removal of $\mathrm{CO}_{2}$ in diabetic fibres.

In addition, a decreased concentration of esterase D formylglutathione hydrolase might cause toxic side effects. The reduced expression of dihydrolipoamide succinyltransferase, the core enzyme of the 2-oxoglutarate dehydrogenase complex in muscle mitochondria that participates in succinylCoA production (45), agrees with the idea of abnormal mitochondrial functioning in diabetes (46). However, other mitochondrial markers such as isocitrate dehydrogenase, cytochrome c oxidase and Coq 9 protein (47) were shown to be increased in GK muscle, possibly representing a compensatory mechanism to improve the oxidative capacity. This would agree with the increased level of B-globulin.

Diabetes seems to have a differential effect on key enzymes of the glycolytic pathway. While enolase levels were shown to be decreased, phosphoglucomutase and aldolase exhibited an elevated concentration in GK muscles. Since many glycolytic enzymes are multi-functional, it is difficult to interpret how insulin resistance triggers these altered expression patterns. However, changed abundance in these enzymes will certainly alter the glycolytic flux in diabetic muscle. Interestingly, pyruvate dehydrogenase, the key linker enzyme that connects glycolysis with the citric acid cycle, is elevated. This might be a compensatory mechanism in glucose-starved diabetic muscle tissues and might help to maximise the transformation of glycolysis-derived pyruvate into acetyl-CoA. In analogy to a recent proteomic survey of obese muscle (48), the AK1 isoform of adenylate kinase is increased in diabetes, suggesting alterations in nucleotide metabolism. Higher concentrations of the contractile elements $\alpha$-actin and the capping protein of the actin filament indicate diabetesdependent repair mechanisms of the thin filament. This would agree with increased levels of stress proteins and anti-oxidant markers, such as the small heat shock protein Hsp27/B1, 3-mercaptopyruvate sulfurtransferase and $\mathrm{Cu} / \mathrm{Zn}$ superoxide dismutase. Their up-regulation demonstrates a considerable need to counter-act cellular damage due to diabetes.

The protein species with the highest fold increase in GK muscle was identified as monoglyceride lipase. This enzyme 
mediates a critical step in the hydrolysis of stored triglycerides (49) and its up-regulation might represent increased energy utilization by the lipolytic pathway in glucose-starved muscle tissues. More detailed biochemical studies have to be carried out to determine the general suitability of monoglyceride lipase as a muscle marker of non-obese diabetes. However, it is clear that insulin resistance results in a lack of glucose uptake by muscle cells, which in turn has an effect on other metabolic pathways such as gluconeogenesis, triacylglycerol hydrolysis, fatty acid oxidation and ketone body formation.

In conclusion, this initial study of changes in the protein expression pattern of GK muscle has identified interesting new candidates for the establishment of a biomarker signature of diabetic skeletal muscle. It will now be critical to correlate these findings to investigations on human skeletal muscle (50-52) and to conduct more detailed studies with other protein dyes that exhibit a different dynamic labelling range, such as fluorescent methodologies (53). In the future, new signature molecules will hopefully be useful for the improvement of diagnostic methods and the identification of superior therapeutic targets to eliminate diabetes-associated muscle weakness.

\section{Acknowledgements}

Research was supported by a project grant from the Irish Health Research Board (HRB-RP/2005/3), a Hume Scholarship from NUI Maynooth and an equipment grant from the Higher Education Authority (HEA-RERGS-07-NUIM). The authors thank Ms. Caroline Batchlor (NUI Maynooth) for assistance with mass spectrometry.

\section{References}

1. King H, Aubert RE and Herman WH: Global burden of diabetes, 1995-2025: prevalence, numerical estimates, and projections. Diabetes Care 21: 1414-1431, 1998.

2. Luna MTT: Genes and type 2 diabetes mellitus. Arch Med Res 36: 210-222, 2005.

3. Scheen AJ: Pathophysiology of type 2 diabetes. Acta Clin Belg 58: 335-341, 2003.

4. Petersen KF and Shulman GI: Pathogenesis of skeletal muscle insulin resistance in type 2 diabetes mellitus. Am J Cardiol 90: G11-G18, 2002.

5. Sander GE and Giles TD: Diabetes mellitus and heart failure. Am Heart Hosp J 1: 273-280, 2003.

6. Andersen H, Nielsen S, Mogensen CE and Jakobsen J: Muscle strength in type 2 diabetes. Diabetes 53: 1543-1548, 2004.

7. Park SW, Goodpaster BH, Strotmeyer ES, de Rekeneire N, Harris TB, Schwartz AV, Tylavsky FA and Newman AB: Decreased muscle strength and quality in older adults with type 2 diabetes. Diabetes 55: 1813-1818, 2006.

8. Phielix E and Mensink M: Type 2 diabetes mellitus and skeletal muscle metabolic function. Physiol Behav 94: 252-258, 2008.

9. Kraegen EW and Cooney GJ: Free fatty acids and skeletal muscle insulin resistance. Curr Opin Lipidol 19: 235-241, 2008.

10. Korc M: Diabetes mellitus in the era of proteomics. Mol Cell Proteomics 2: 399-404, 2003.

11. Sundsten T and Ortsaeter H: Proteomics in diabetes research. Mol Cell Endocrinol 297: 93-103, 2009.

12. Kitahara A, Toyota T, Kakizaki M and Goto Y: Activities of hepatic enzymes in spontaneous diabetes rats produced by selective breeding of normal Wistar rats. Tohoku J Exp Med 126: 7-11, 1978 .

13. Abdel-Halim SM, Guenifi A, Luthman H, Grill V, Efendic S and Ostenson CG: Impact of diabetic inheritance on glucose tolerance and insulin secretion in spontaneously diabetic GKWistar rats. Diabetes 43: 281-288, 1994.
14. Krook A, Kawano Y, Song XM, Efendic S, Roth RA, WallbergHenriksson $\mathrm{H}$ and Zierath JR: Improved glucose tolerance restores insulin-stimulated Akt kinase activity and glucose transport in skeletal muscle from diabetic Goto-Kakizaki rats. Diabetes 46: 2110-2114, 1997.

15. Portha B, Serradas P, Bailbe D, Suzuki K, Goto Y and Giroix MH: Beta-cell insensitivity to glucose in the GK rat, a spontaneous nonobese model for type II diabetes. Diabetes 40: 486-491, 1991.

16. Witte K, Jacke K, Stahrenberg R, Arlt G, Reitenbach I, Schilling L and Lemmer B: Dysfunction of soluble guanylyl cyclase in aorta and kidney of Goto-Kakizaki rats: influence of age and diabetic state. Nitric Oxide 6: 85-95, 2002.

17. Mulvey C, Harno E, Keenan A and Ohlendieck K: Expression of the skeletal muscle dystrophin-dystroglycan complex and syntrophin-nitric oxide synthase complex is severely affected in the type 2 diabetic Goto-Kakizaki rat. Eur J Cell Biol 84: $867-883,2005$.

18. Mulvey C, Mullen E and Ohlendieck K: The pathobiochemical role of the dystrophin-dystroglycan complex and the $\mathrm{Ca}^{2+}$ handling apparatus in diabetes-related muscle weakness (review). Mol Med Rep 1: 297-306, 2008.

19. Dadke SS, Li HC, Kusari AB, Begum N and Kusari J: Elevated expression and activity of protein-tyrosine phosphatase $1 \mathrm{~B}$ in skeletal muscle of insulin-resistant type II diabetic GotoKakizaki rats. Biochem Biophys Res Commun 274: 583-589, 2000.

20. Steiler TL, Galuska D, Leng Y, Chibalin AV, Gilbert M and Zierath JR: Effect of hyperglycemia on signal transduction in skeletal muscle from diabetic Goto-Kakizaki rats. Endocrinology 144: 5259-5267, 2003.

21. Shen W, Hao J, Tian C, Ren J, Yang L, Li X, Luo C, Cotma CW and Liu J: A Combination of nutriments improves mitochondrial biogenesis and function in skeletal muscle of type 2 diabetic Goto-Kakizaki rats. PLoS ONE 3: E2328, 2008.

22. Yasuda K, Nishikawa W, Iwanaka N, Nakamura E, Seino Y, Tsuda $\mathrm{K}$ and Ishihara A: Abnormality in fibre type distribution of soleus and plantaris muscles in non-obese diabetic GotoKakizaki rats. Clin Exp Pharmacol Physiol 29: 1001-1008, 2002.

23. Doran P, Donoghue P, O'Connell K, Gannon J and Ohlendieck K: Proteomic profiling of pathological and aged skeletal muscle fibres by peptide mass fingerprinting (review). Int J Mol Med 19: 547-564, 2007.

24. Doran P, Gannon J, O'Connell K and Ohlendieck K: Proteomic profiling of animal models mimicking skeletal muscle disorders. Proteomics Clin Appl 1: 1169-1184, 2007.

25. Doran P, Martin G, Dowling P, Jockusch H and Ohlendieck K: Proteome analysis of the dystrophin-deficient MDX diaphragm reveals a drastic increase in the heat shock protein cvHSP. Proteomics 6: 4610-4621, 2006.

26. Bradford MM: A rapid and sensitive method for the quantitation of microgram quantities of protein utilizing the principle of protein-dye binding. Anal Biochem 72: 248-254, 1976.

27. Donoghue P, Doran P, Wynne K, Pedersen K, Dunn MJ and Ohlendieck K: Proteomic profiling of chronic low-frequency stimulated fast muscle. Proteomics 7: 3417-3430, 2007.

28. Doran P, O'Connell K, Gannon J, Kavanagh M and Ohlendieck K: Opposite pathobiochemical fate of pyruvate kinase and adenylate kinase in aged rat skeletal muscle as revealed by proteomic DIGE analysis. Proteomics 8: 364-377, 2008.

29. Neuhoff V, Arold N, Taube D and Ehrhardt W: Improved staining of proteins in polyacrylamide gels including isoelectric focusing gels with clear background at nanogram sensitivity using Coomassie Brilliant Blue G-250 and R-250. Electrophoresis 9: 255-262, 1988.

30. Heukeshoven J and Dernick R: Simplified method for silver staining of proteins in polyacrylamide gels and the mechanism of silver staining. Electrophoresis 6: 103-112, 1985.

31. Rabilloud T, Strub JM, Luche S, van Dorsselaer A and Lunardi J: A comparison between Sypro Ruby and ruthenium II tris (bathophenanthroline disulfonate) as fluorescent stains for protein detection in gels. Proteomics 1: 699-704, 2001.

32. Staples GO, Bowman MJ, Costello CE, Hitchcock AM, Lau JM, Leymarie N, Miller C, Naimy H, Shi X and Zaia J: A chip-based amide-HILIC LC/MS platform for glycosaminoglycan glycomics profiling. Proteomics 9: 686-695, 2009.

33. Gannon J, Doran P, Kirwan A and Ohlendieck K: Drastic increase of myosin light chain MLC-2 in senescent skeletal muscle indicates fast-to-slow fibre transition in sarcopenia of old age. Eur J Cell Biol 88: 685-700, 2009. 
34. Okumura N, Hashida-Okumura A, Kita K, Matsubae M, Takao T and Nagai K: Proteomic analysis of slow- and fast-twitch skeletal muscles. Proteomics 5: 2896-2906, 2005.

35. Gelfi C, Vigano A, De Palma S, Ripamonti M, Begum S Cerretelli $\mathrm{P}$ and Wait R: 2-D protein maps of rat gastrocnemius and soleus muscles: a tool for muscle plasticity assessment Proteomics 6: 321-340, 2006.

36. O'Connell K, Gannon J, Doran P and Ohlendieck K: Proteomic profiling reveals a severely perturbed protein expression pattern in aged skeletal muscle. Int J Mol Med 20: 145-153, 2007.

37. Gannon J, Staunton L, O'Connell K, Doran P and Ohlendieck K: Phosphoproteomic analysis of aged skeletal muscle. Int $\mathrm{J}$ Mol Med 22: 33-42, 2008.

38. Yan JX, Harry RA, Wait R, Welson SY, Emery PW, Preedy VR and Dunn MJ: Separation and identification of rat skeletal muscle proteins using two-dimensional gel electrophoresis and mass spectrometry. Proteomics 1: 424-434, 2001.

39. Piec I, Listrat A, Alliot J, Chambon C, Taylor RG and Bechet D: Differential proteome analysis of aging in rat skeletal muscle. FASEB J 19: 1143-1145, 2005.

40. Doran P, Gannon J, O'Connell K and Ohlendieck K: Aging skeletal muscle shows a drastic increase in the small heat shock proteins $\alpha \mathrm{B}$-crystallin/HspB5 and cvHsp/HspB7. Eur J Cell Biol 86: 629-640, 2007.

41. Zimmet P, Alberti KG and Shaw J: Global and societal implications of the diabetes epidemic. Nature 414: 782-787, 2001.

42. Gregg EW, Beckles GL, Williamson DF, Leveille SG Langlois JA, Engelgau MM and Narayan KM: Diabetes and physical disability among US adults. Diabetes Care 23: 61-67, 2000 .

43. Murin R, Schaer A, Kowwtharapu BS, Verieysdonk S and Hamprecht B: Expression of 3-hydroxy-isobutyrate dehydrogenase in cultured neural cells. J Neurochem 105: 1176-1186, 2008.

44. Geers C and Gros G: Carbon dioxide transport and carbonic anhydrase in blood and muscle. Physiol Rev 80: 681-715, 2000.
45. Matuda S, Kodama J, Goshi N, Takase C, Nakano K, Nakagawa S and Ohta S: A polypeptide derived from mitochondrial dihydrolipoamide succinyltransferase is located on the plasma membrane of skeletal muscle. Biochem Biophys Res Comm 241: 151-156, 1997.

46. Mogensen M, Sahlin K, Fernstroem M, Glintborg D, Vind BF Beck-Nielsen $\mathrm{H}$ and Hojlund $\mathrm{K}$ : Mitochondrial respiration is increased in skeletal muscle of patients with type 2 diabetes. Diabetes 56: 1592-1599, 2007.

47. Sohal RS and Forster MJ: Coenzyme Q, oxidative stress and aging. Mitochondrion 7: S103-S111, 2007.

48. Hittel DS, Hathout Y, Hoffman EP and Houmard JA: Proteome analysis of skeletal muscle from obese and morbidly obese women. Diabetes 54: 1283-1288, 2005.

49. Zechner R, Kienesberger PC, Haemmerle G, Zimmermann R and Lass A: Adipose triglyceride lipase and the lipolytic catabolism of cellular fat stores. J Lipid Res 50: 3-21, 2009.

50. Hojlund K, Wrzesinski K, Larsen PM, Fey SJ, Roepstorff P, Handberg A, Dela F, Vinten J, McCormack JG, Reynet C and Beck-Nielsen $\mathrm{H}$ : Proteome analysis reveals phosphorylation of ATP synthase beta-subunit in human skeletal muscle and proteins with potential roles in type 2 diabetes. J Biol Chem 278: 10436-10442, 2003.

51. Stentz FB and Kitabchi AE: Transcriptome and proteome expressions involved in insulin resistance in muscle and activated T-lymphocytes of patients with type 2 diabetes. Genomics Proteomics Bioinformatics 5: 216-235, 2007.

52. Hwang H, Bowen BP, Lefort N, Flynn CR, De Filippis EA, Roberts C, Smoke CC, Meyer C, Hojlund K, Yi Z and Mandarino LJ: Proteomics analysis of human skeletal muscle reveals novel abnormalities in obesity and type 2 diabetes mellitus. Diabetes (In press).

53. Viswanathan S, Unlu M and Minden JS: Two-dimensional difference gel electrophoresis. Nat Protoc 1: 1351-1358, 2006. 